\title{
Integrating Industry Seminars within a Software Engineering Module to Enhance Student Motivation
}

\author{
G.J. Collins \\ University College London/School of Management, London, United Kingdom \\ g.collins@ucl.ac.uk
}

\begin{abstract}
Engineering students increasingly require and demand coverage of emerging technologies to prepare themselves for subsequent research and employment. Industry and professional bodies are also concerned that engineering education doesn't always prepare students adequately for the world of work.
\end{abstract}

The software engineering postgraduate professional practice module at University College London is designed to provide real-world experience, before students commence their industry research projects. Industry speakers are invited from a range of organizations, including ThoughtWorks, IBM, Form3, Verne Global, and Fujitsu.

Seminars include: DevOps, microservices, cloud-native architectures, machine learning and quantum technologies. Before each topic is covered, students are asked their understanding of the subject matter, via questionnaires. This information is shared with industry speakers to ensure the content of presentations is compatible with students' prior knowledge. It has proved valuable to allow time for discussions to facilitate professional networking, which particularly benefits female students.

Students have indicated they highly value the real-world project examples delivered by industry experts. This suggests that integrating industry seminars can enhance engineering education and motivate students by covering leading-edge technologies and practices. However, this requires considerable time in coordinating and codeveloping seminars, and such initiatives need to be adequately resourced to be effective.

Keywords - engineering education; professional networking; industry seminars; motivation

\section{INTRODUCTION}

There is shortfall of engineers in the UK; this problem of recruiting qualified engineers and researchers extends across Europe [1, 2]. One way to improve this situation is to ensure university teaching better equips students for the demands of industry. Another is to deliver topics that students have indicated they want to study; to help improve retention and increase applications from those less represented in society.
The Royal Academy of Engineering (RAEng) recommends to reduce this shortfall of engineers there should be increased industry-academic engagement [3]. They outline that industry involvement in teaching provides real-world examples and can increase motivation in the subject, by highlighting relevance and practical examples. The RAEng also recommend that project management skills are taught as an integral part of engineering courses, delivered by business school staff within universities. This is the case at University College London (UCL), where the professional practice lectures are taught by a member of staff based in the UCL School of Management.

Fung [4] argues that there should be no conflict between the needs of industry and that of university research. Both require inquiry and critical thinking. UCL's Connected Curriculum [5] instills that students learn more effectively through research and inquiry. One approach to inculcate this philosophy is not only integrating research within the curriculum but to integrate research and development project examples via guest speakers from industry.

\section{MODULE DESIGN}

The software engineering postgraduate professional practice module is delivered within the Department of Computer Science, which is a division of the Faculty of Engineering Sciences. The module is designed as a series of 10, 2-hour, afternoon industry seminars, with a project management lecture and tutorial of 2 hours the following day. Students study this module during the spring term, immediately before commencing their research projects.

This module provides an opportunity to cover projects that all students have expressed an interest in. Male students have mentioned delivery pipelines and technical approaches. Female students have outlined interests particularly in health and sustainability [6]. Increasingly, students have mentioned over the years an interest in research that has a positive impact on the environment. These topics provide an ideal opportunity to show the relationship with the UN's sustainability goals. 
The module design places emphasis on considering a wide range of users and their views to improve solutions. Discussing previous students' research projects is a way to show the different approaches students have taken and reinforces the need to consider the different perspectives of stakeholders. An initial lecture on inclusion and diversity also highlights the importance of neurodiversity: different ways of thinking and communicating about a problem. The module aims to foster inclusion and sustainability throughout. It is aligned to the Engineering and Physical Sciences Research Council's (EPSRC's) Framework for Responsible Innovation [7] which is also embedded in the Horizon 2020 initiatives for inclusive and sustainable research and innovation [8]. The teaching includes the EPRSc's Anticipate, Reflect, Engage and Act (AREA) framework, to ensure students continually evaluate the ethical and societal impact of their research projects. The UCL Department of Computer Science has received the highest category within the UK university sector Athena SWAN (Scientific Women's Academic Network) award; recognition for the promotion of diversity and furthering research opportunities and careers for women engineers [9].

By integrating the project management lectures with the industry seminars, the aim is to create a cohesive module. The project management lectures include case studies and outline previous student group research projects. Students are keen to see what previous cohorts of students have achieved in their research projects, and the environmental and societal impact these have made. The initial examples cover student research projects using machine learning to reduce air pollution exposure for pedestrians and cyclists [10]. They demonstrate how students have elaborated goals, planned their research, and provide a way to highlight further research opportunities. The aim is also to widen the knowledge of the students to help create what some have called "T-Shaped engineers" [11], where they not only understand the technical aspects, but are better able to manage projects, and appreciate the wider implications of their research for society.

\section{ENSURING STUDENT MOTIVATION}

By establishing students' understanding of topics via questionnaires, before coverage within the module, seminars can be better tailored to the students' needs.

As an example, Fig. 1., outlines a question before the architecture topic is delivered. This is not to catch students out but to consider what in their opinion is the most appropriate answer. The answers to these questions formed a basis for discussions, for the project management and technical challenges. These discussions were continued within their electronic module forum using Moodle.

For the question "How likely are you to document architecture decisions" Fig. 1. the choices were:
1. Although there are some industry approaches regarding architecture decisions I would not necessarily look at these, but would at least document the problem, a solution. and an alternative.

2. With further help or working as a team I feel confident I could adapt an appropriate method, document a solution, outline an alternative and describe why our chosen solution was adopted.

3. I am aware of Decision Centric Architecture Reviews (DCAR) or an alternative method and may reference this and adapt this method so that the time involved in using this is not an overhead to the project. As well as the solution and an alternative I may include forces in favor of our solution as well as those against.

Students are likely to give answers aligned to their own way of thinking and their own viewpoint. Students are also less likely to feel defensive over their answers, than if they were asked after a teaching session [12]. Explaining the purpose to students of these questionnaires also helps to build a shared endeavor of learning, in that they can appreciate that the teaching material is being tailored to their understanding and their future research and employment needs.

The answers to these questions and follow-on discussions helped determine what background material the industry speaker would need to cover. They also help tailor the notes for the project management lectures. Tailoring the content to students' answers ensures that leading edge examples are incorporated and that well understood concepts are not repeated.

By discussing these answers and the proposed project management content with the industry speaker, this ensures a more consistent structure for the module.

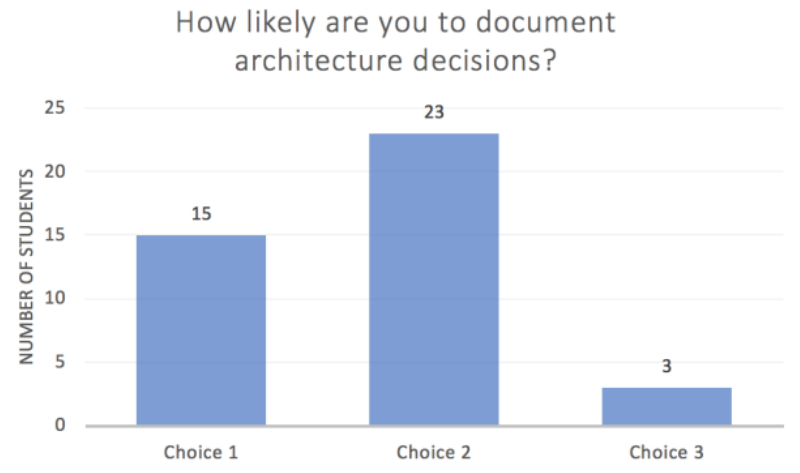

Figure 1. Answers provided by the students attending the professional practice module academic year 2017-18. Example shows the results to one section of the questionnaire, for the topic architecture, covering architecture decisions. Participants $=41$. Class size 44 , including 5 students from the UCL School of Management. 15 students opted for choice 1, 23 opted for choice 2, and 3 for choice 3 . 
By sharing this information with the industry speakers, they also have a better understanding of the level they need to aim at, and are more likely to feel they are working as a team with the academic staff. This builds a better basis for creating a more coherent vision for the module and building relationships that may lead on to joint university and industry research collaborations.

The industry seminars cover current research and development projects and are designed to help students when they start their research projects. The topics are designed to cover project management as well as technical issues.

The industry seminars academic year 2018/19 were from speakers from a range of organizations:

1. HSBC, Advances in (High Performance Computing) HPC for exposure analytics

2. Verne Global, HPC for AI applications and sustainable data center design

3. NetApp, Building the Hybrid Cloud, DevOps: Agility without sacrificing enterprise stability

4. Fujitsu, Quantum Computing

5. Form3, Evolving Systems Design: from unreliable (Remote Procedure Call) RPC to resilience using Linkerd. Coverage of containers and schedulers and microservice architecture

6. Instana, Producing Actionable Information from Monitoring Data. Automatic Agents, covering detection algorithms, neural networks, and dynamic graphs

7. RedHat, Containers, Kubernetes, OpenShift and OpenStack

8. IBM, Information Architecture, Delivering Innovation

9. Digital Asset, Making Contracts Smart. Integration of business logic within the distributed ledger

10. ThoughtWorks, Microservices, NGINX, Microservices, demonstration of a denial-ofservice attack (DoS attack)

There are also one-hour tutorials to supplement the industry seminars and project management lectures to encourage students to discuss in more detail the issues raised in both sessions. Project management sessions cover project examples and case studies, using a "flipped lecture" [13] approach, to create an active learning opportunity, by devoting class time to analyzing and evaluating the information. The scenario and background information are provided to students online before the class, leaving class time to discuss the material in detail.

Project management lectures 2018/2019 delivered by UCL School of Management lecturer:

1. Introduction, including student air pollution reduction projects using machine learning
2. Case study of a software engineering project

3. Agile project management

4. Retrospectives and reviews, using project examples

5. Boundary communications case study

6. Software architecture decisions, multi-objective optimization

7. Risk and opportunity, use of machine learning in Artificial Intelligent Operations (AIOps)

8. Scheduling practical exercise

9. Teamwork, inclusion, and diversity

10. Sustainable IT. Process improvement at scale and within agile development projects.

Industry seminars are interlinked with the project management lectures. For example, one seminar illustrates how machine learning can be used to identify problems earlier within software development cycle. These concepts are reinforced within the project management stream showing how machine learning can be used to reduce alerts, which can cause fatigue for engineers responsible for software delivery pipeline, and how this time can instead be used for more creative work. Students' research projects, which use machine learning to establish lower pollution routes for pedestrians are outlined as well. Related examples are also covered showing how AI can be applied to provide optimal routes for distribution during disaster relief. Other examples highlight the benefits within medicine; how machine learning can provide a higher level of accuracy in diagnosis within cancer screening programs.

At every stage in planning the module there is a reflection on how the content will engage and motivate the class. It is argued that the way the instructor frames the work is a major factor for student motivation [14]. To inspire students, introductory topics are based on previous students' research projects and how these have made a positive impact on society. To motivate the class further examples of how students during the previous academic year have carried their research forward within industry and research programs at UCL are also highlighted.

\section{Challenges}

Sourcing inspiring speakers takes considerable time. Students have outlined they particularly appreciate speakers that demonstrate interactive examples. The industry speakers are selected for their enthusiasm for their subject and their ability to answer technical questions clearly. Invariably, this means that the speakers are passionate about their work and committed to helping their teams pioneer advances in research. To improve the module students are encouraged during the final week of the module, to complete an anonymous online feedback form, within their own time.

"The lecturer managed to secure talks from incredible industry speakers who shared up-to-date, relevant 
knowledge on current topics" Professional practice student 2017-18

The students find seminars with live coding particularly engaging; the NGINX seminar included an example of the prevention of a denial of service attack (DoS attack):

"I especially appreciated the talks from NGINX and Chef, which were two companies with whose products I'm intimately familiar with." Professional practice student 2017-18

The industry speaker from NGINX specifically created a website, so that the provision of this software could be demonstrated. Here after outlining security concepts a volunteer from the audience was asked to initiate a DoS attack. As students have as their main interest software engineering, it was not surprising that talks that had live coding examples were the most popular (c.f. modern interactive museum exhibits).

They also appreciated the relevance to their forthcoming research projects and software development careers.

"The speakers where very interesting. Many of the topics touched on in the project management part were important for our future careers..."

"[The lecturer] finds ways to motivate student participation and he finds amazing guest speakers from industry."

\section{Professional Practice students 2018/19}

However, one student mentioned within the anonymous feedback 2017/18 and again 2018/19 that having a written assessed component for this module would be a better approach, rather than rely fully on the examination.

"I think having the lecturers from industry was both very interesting and really useful. Some of those lectures have been my highlights at UCL. Maybe an assignment that allows students to select 3 of the visiting talks then to write a short report... That way you would need a 100\% exam."

"I believe that an exam is not a good way to asses this module...I think coursework would be more appropriate as it would take a longer time to put thought into the valuable topics and find references and supporting claims."

Professional Practice students 2017-2018 and 2018/19 respectively.

This feedback is being taken in to consideration in redesigning the assessment of the module for future years, to include assignments as well as a final examination. The assessment of the students' research projects is based on their research approach, reports, and presentations within their follow-on module.

\section{PlanNING SEMINARS}

Researchers have recognized that collaborating to build effective relationships with industry speakers is time consuming [15]. When inviting an industry speaker, a follow-up meeting is necessary, either at the university or more usually at their software development site, to discuss coding practices and the proposed seminar. An outline of the module is provided to the industry speaker, with a summary of main objectives and that students are keen to understand real-world scenarios and the dilemmas teams may face. Industry speakers need to be aware of the students' skill level and languages they use for coding. There also needs to be a discussion how their topic interrelates to the other modules and research at UCL. Reviews of the slides and content occur well in advance of the industry seminar. These regular communications, Fig. 2., also help avert any problems, as industry speakers are more likely to feel part of the team, delivering the common objectives of the research module.

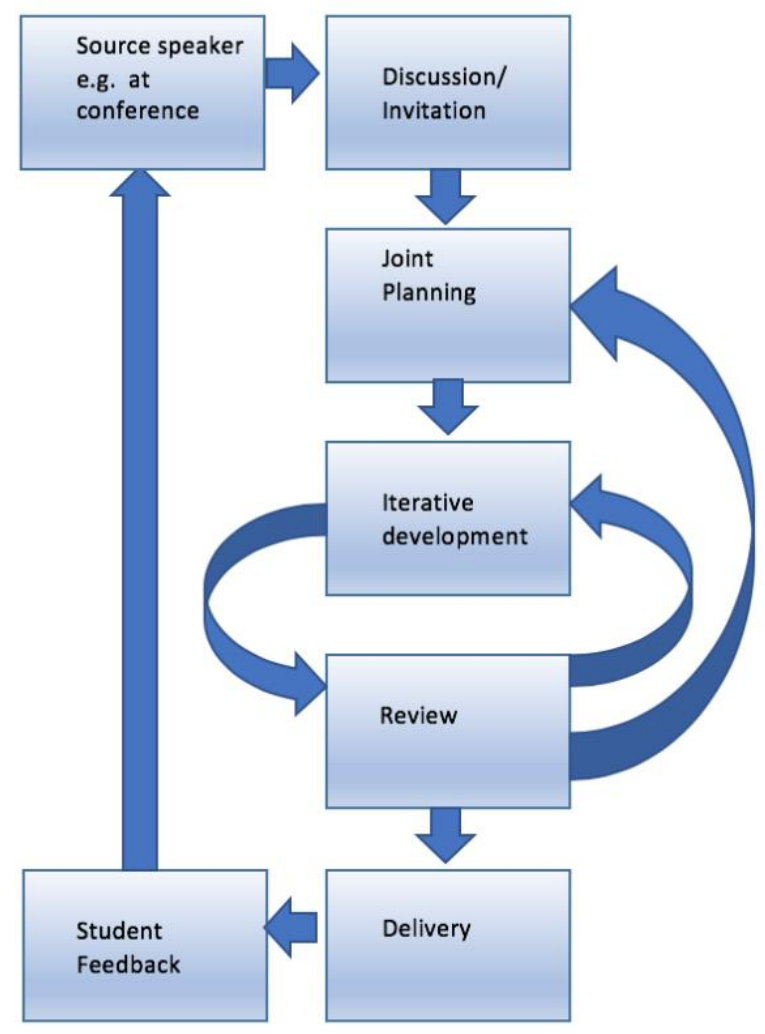

Figure 2. The stages of planning and iterative development of industry seminars for the UCL professional practice module. The review process involving the industry speaker and academic feeds into the development of the seminar and future planning. Student feedback determines future topics. 


\section{DISCUSSION}

Business-university research collaborations provide significant benefits to their participants. Yet from the Dowling report [16] it seems that industry or universities are not reaping the full benefits of these collaborations. Key to these collaborations are the people involved and building long lasting and trusted relationships. These require a mutual understanding of the goals of both sides and a willingness to collaborate to achieve a successful common vision. To facilitate this enough time needs to be allocated for those involved within their work schedules.

The advantages of integrating industry speakers are that they provide a real-world perspective and frequently opportunities for research. Industry speakers often invite students and staff to forthcoming conferences on a complementary basis. The research students regularly accept and value these invitations, which due to budget limitations they may not otherwise be able to attend. Speakers also outline any further opportunities to contact them and their organization, or visits to see their development and research environment. Having this period of discussion, which breaks down the initial barriers of communication, may be one approach that can help students develop their professional networks.

Davey [17] suggests that there are two important aspects to professional networks the instrumental, the assistance and advice, and the psychosocial including social aspects. Instrumental support can also be considered as tangible support [18], actions that are useful for career development. Instrumental networking, the formation of ties with others explicitly for professional goals, are important for cooperation at work as well as career development. Students often have well developed social networks but limited professional networks. By providing adequate time for discussions with industry speakers this provides an opportunity for students to develop their professional networks. This is particularly important for female students who are less likely to have the support of professional networks [19]. However, previous research by the author has outlined that female students are keen to engage with industry if given the opportunity [6]. Casciaro et al. have shown [19] that females often perceive that instrumental networking can be a morally compromising, by creating an unfair career advantage for themselves. This research has shown that facilitating such networking opportunities, these barriers which often limit female students in their careers, may be broken down. By providing the opportunity at the end of the speaker seminars and speakers initiating the networking and the further work visits, this may be one way that academic modules can support all students, and address the imbalance reported in female professional networking.

Having different perspectives outlined also helps students appreciate the need to engage and listen to their colleagues and the different views within their teams. Within the project management lectures the importance of this process is emphasized. If, for example, during a technical discussion the team eventually reject an idea, then the team member who proposed this idea at least feels as though their views are listened to, and if discussed in detail will also understand the reasons why. This is reinforced during the lecture covering architecture decisions. Here the method of proposing an architectural solution, the pros and cons are recorded, and any methods accepted or rejected are also explained.

How industry speakers are integrated effectively in delivering engineering modules is an area of research that needs to be addressed. May-Newman and Cornwall [20] outline problems where an industry speaker does not have the pedagogic experience, or where the academic does not have the practical experience. Conferences are an essential way to keep up to date but are also a rich source of speakers who can communicate effectively. Collaborating and co-developing seminars resolves the latter issue; it also ensures coverage of the syllabus. By integrating industry speakers within a module this addresses the problem of the academic not having practical experience; and a range of speakers addresses that a real-world expert delivers every topic. After all, it cannot be expected that a lecturer is an expert in every area.

Research has shown that inviting and collaborating with industry speakers is time consuming. Fung [4] has pointed out this should be adequately resourced. This is emphasized by the RAEng that this should be more a joint venture. However, experience reports [15] indicate that this activity is often left to one lecturer in a module. Having a single point of contact is a risk; a lecturer may be absent or may be assigned to another role. Whether these industry speaker invitations should be a centrally organized or not is interpreted differently within departments in the UCL Engineering Sciences faculty. It however needs to be recognized that often a speaker will provide a seminar as they have a professional relationship to the academic that has first invited them. The problem with communicating solely through a central system is this professional relationship may be lost if the original party is not involved.

\section{CONCLUSIONS}

Strategic links between universities and industry provide myriad benefits. For universities, the staff and students, opportunities to address real-world applications and challenging research problems. This provides an opportunity for leading edge research opportunities for students. Industry-into-academia initiatives can further enhance this, via visiting professorships and visiting speakers. The faculty of Engineering Sciences at UCL benefit from both aspects. This can also provide a conduit 
for ad hoc mentoring and career advice. This is further supported by providing additional time after industry seminars for further questions and discussion. This activity seems to particularly support those less represented in the engineering profession to engage in networking.

Sharing the information prior to seminars and feedback from students is essential in developing seminars at the appropriate level. Also, it a way to help speakers feel they are part of a shared process in developing the skills and knowledge of future engineers. The closer the collaboration, the more likely there will be successful research academic-industry opportunities identified in the future, for both research staff and students. Another positive outcome is the practical experience provided to staff and students for the visits as well as the career networking opportunities. In addition to this are the events and conferences that staff and students are invited to, often on a complementary basis. Sharing the knowledge helps also to deliver the ethos of the connected curriculum; integrating teaching and research. It also provides a valuable example to students of academic-industry collaboration, something we are trying to encourage within their research projects.

The industry speakers within the module provide a wide range of perspectives. This reinforces the idea that within teams, workshops and in projects we need to consider different views and perspectives. It also helps students understand that diversity in teams is an asset if we wish to create solutions for the whole of society. If we wish that students develop engineering solutions for the benefit of society then we need support from industry to help develop students' research experiences.

Integrating industry speakers within engineering research modules has been shown to help prepare students for their forthcoming research projects as well as world of work. The students' feedback from this module is that industry speakers are highly valued and provide an important adjunct to the project examples and technology coverage. However, adequate time needs to be allocated for this to be effective. Senior managers need to be aware of the considerable development time required. There are the overheads of sourcing speakers, understanding in detail what students require, planning and co-developing the seminars, and reviewing the material. The rewards for students of well-designed modules are immense; the increase in motivation and preparation for their research projects as well as increased networking opportunities. With humanity facing ever more complex problems we need to ensure that students have the best preparation for research. If students are introduced to real-world problems and dilemmas within their research studies this will provide a better basis for them to solve the increasingly complex engineering problems and help them create a more sustainable future.

\section{ACKNOWLEDGMENT}

The author acknowledges the kind support of colleagues, Nicolas Gold and Emmanuel Letier, contributing industry speakers, and the UCL software engineering professional practice research students.

\section{REFERENCES}

[1] https://www.randstad.co.uk/career-advice/skills/uk-engineeringfacing-skills-crisis-where-are-the-jobs/

[2] Harvey Nash/KPMG CIO Survey 2019 https://www.hnkpmgciosurvey.com/charts/home.php

[3] Effective Industrial Engagement in Engineering Education https://www.raeng.org.uk/publications/reports/effective-industrialengagement-in-engineering-edu

[4] D. Fung, A Connected Curriculum for Higher Education, UCL Press, 2017.

[5] https://www.ucl.ac.uk/teaching-learning/connected-curriculumframework-research-based-education

[6] G. Collins, "Challenges for teaching sustainability and promoting diversity within a software engineering course," in Proceedings of the $45^{\text {th }}$ European Society for Engineering Education (SEFI) Conference, 18-21 September 2017, Azores, Portugal, pp. 41-48. https://www.sefi.be/conference/2017-azores/

[7] https://epsrc.ukri.org/research/framework/

[8] https://ec.europa.eu/programmes/horizon2020/en/h2020section/responsible-research-innovation

[9] https://www.ucl.ac.uk/computer-science/about/women-computerscience

[10] G. Collins, H. Varilly, and T. Yoshinori, "Pedagogical lessons from an international collaborative big data undergraduate research project," in Proceedings of the 2015 European Conference on Software Architecture Workshops (ECSAW'15), ACM, New York, Article 32, pp. 1-6.

[11] D. L. Johnstone, "Scientists Become Managers - The T-Shaped Man," IEEE Engineering Management Review, Vol. 6 (3), pp. 67 68, 1978 .

[12] M. Goldsmith, "Try feedforward instead of feedback," The Journal for Quality and Participation, vol. 26 (3), pp. 38-40, 2003.

[13] J. Novak, B. Kensington-Miller, T. Evans, "Flip or flop? Students' perspectives of a flipped lecture in mathematics," International Journal of Mathematical Education in Science and Technology, vol. 48(5), pp. 647-658, 2017.

[14] M. Vansteenkiste, J. Simons, W. Lens, K.M. Sheldon, and E. L. Deci, "Motivating learning, performance, and persistence: the synergistic effects of intrinsic goal contents and autonomysupportive contexts," Journal of Personality and Social Psychology, vol 87(2), pp. 246-260, 2004.

[15] C. Armatas and T. Papadopoulos, "Approaches to work integarted learning and engaging industry in vocational ICT courses: evaluation of an Australian pilot program," International Journal of Training Research, vol. 11(1), pp. 56-68, 2013.

[16] https://www.raeng.org.uk/publications/reports/the-dowlingreview-of-business-university-research

[17] L. Davey, "Everyone's Network Should Provide Two Things," Harvard Business Review, 30 ${ }^{\text {th }}$ September 2016.

[18] N. K. Semmer, et al., "The Emotional Meaning of Instrumental Social Support," International Journal of Stress Management, vol 15(3), pp. 235-251, 2008.

[19] T. Casciaro, F. Gino, and M. Kouchaki, "The contaminating effects of building instrumental ties: how networking can make us feel dirty," Administartive Science Quarterly, vol. 59 (4), pp. 705 735, 2014.

[20] K. May-Newman and G. B. Cornwall, "Teaching medical device design using design control," Expert Review of Medical Devices, vol. 9(1), pp.7-14, 2012. 